\title{
Peripheral nerve blocks versus general anesthesia for total knee replacement in elderly patients on the postoperative quality of recovery
}

This article was published in the following Dove Press journal:

Clinical Interventions in Aging

18 February 2014

Number of times this article has been viewed

\author{
JunLe Liu',* \\ WeiXiu Yuan ${ }^{1, *}$ \\ XiaoLin Wang ${ }^{1, *}$ \\ Colin F Royse ${ }^{2,3}$ \\ MaoWei Gong' \\ Ying Zhao' \\ Hong Zhang'
}

'Anesthesia and Operation Center, Chinese People's Liberation Army General Hospital and Medical School of Chinese People's Liberation Army, Beijing, People's Republic of China; ${ }^{2}$ Anesthesia and Pain Management Unit, Department of Surgery, University of Melbourne, Melbourne, Victoria, Australia; ${ }^{3}$ Department of Anesthesia and Pain Management, The Royal Melbourne Hospital, Melbourne, Victoria, Australia

*These authors contributed equally to this work
Correspondence: Hong Zhang

Anesthesia and Operation Center,

Chinese People's Liberation Army

General Hospital, 28 Fuxing Road, Beijing,

100853, People's Republic of China

Tel +86 I0 6693805 I

Fax +86 I0 $8827 \quad 1427$

Email mazuimao30I@I63.com
Background: Both peripheral nerve blocks with sedation or general anesthesia can be used for total knee replacement surgery.

Objectives: We compared these anesthetic techniques on the postoperative quality of recovery early in elderly patients.

Materials and methods: In our study, 213 patients who were $\geq 65$ years old and undergoing total knee replacement were randomized to peripheral nerve blocks (PNBs) - lumbar plexus and sciatic - with propofol sedation, or general anesthesia with combined propofol and remifentanil. Blocks were performed using nerve stimulation and $0.35 \%$ ropivacaine. All patients received postoperative multimodal analgesia. Postoperative recovery was assessed at 15 minutes, 40 minutes, 1 day, 3 days, and 7 days after surgery, with the Postoperative Quality of Recovery Scale, in physiological, nociceptive, emotive, modified activities of daily living, modified cognitive, and overall patient perspective domains.

Results: Intraoperative blood pressure and heart rate were more stable with PNBs $(P<0.001)$. The recovery was better with PNBs in physiological $(P<0.001)$, emotive (depression and anxiety) $(P<0.001)$, nociceptive (pain and nausea) $(P<0.001)$, modified cognitive $(P<0.001)$, and all domains recovery $(P<0.001)$, but not in activities of daily living $(P=0.181)$. Intraoperative drugs and the postoperative sulfentanil requirement of the PNBs group were lower (all $P<0.001$ ). Differences were greatest early after surgery with equivalence by 1 week. Satisfaction was high and not different between groups $(P=0.059)$.

Conclusion: Lumbar plexus and sciatic blocks with sedation facilitates faster postoperative recovery than general anesthesia, but not at 1 week after total knee replacement in patients who were 65 years or older. The trial has been registered at ClinicalTrials.gov. (NCT01871012).

Keywords: nerve block, general anesthesia, knee replacement, perioperative care

\section{Introduction}

Total knee replacement (TKR) is a common and effective treatment for end-stage knee arthritis. As the population ages, the frequency of surgery is increasing proportionally, ${ }^{1}$ bringing with it the challenge of managing older patients with frequent comorbid diseases and an increased risk of complications. Poor postoperative quality of recovery may result in patient and family suffering, a prolonged hospital stay, and a greater demand on health care resources. ${ }^{2,3}$

Both general anesthesia (GA) and regional anesthesia have been used for TKR surgery. Peripheral nerve blocks (PNBs) combining lumbar plexus and sciatic nerve blocks can be used as the primary anesthetic for TKR, or combined with general anesthesia to provide postoperative pain relief. ${ }^{4-6}$ They form an alternative approach 
to the central neuraxial blockade for regional anesthesia for knee surgery and have shown improved postoperative recovery. ${ }^{7}$ This technique has become common in our institution for knee surgery. The quality of recovery is becoming a clinical and research area of increasing significance. It reflects a change in emphasis from hospital-based outcomes to patient-focused outcomes. An early measurement of quality of recovery tends to focus on physiological or nociceptive recovery and readiness for patient discharge; whereas, longer-term recovery tends to focus on patient satisfaction, a poor metric of quality of recovery in objectively measured domains. ${ }^{8}$

The Postoperative Quality of Recovery Scale (PQRS) is a research tool designed to measure the postoperative quality of recovery in multiple domains and over multiple time periods. Recovery domains include physiological, emotive (depression and anxiety), nociceptive (pain and nausea), cognitive, activities of daily living (ADL), and overall patient perspective (including satisfaction). ${ }^{8,9}$ Importantly, the PQRS surveys are objective and measure recovery at predetermined time points, rather than relying on subjective patient recall. Further, the measurement of recovery in multiple domains facilitates an understanding of how recovery in one area can impact on other recovery domains. The inclusion of cognition is important as poor cognitive recovery is being increasingly recognized as a major morbidity.

Our aim was to identify whether our technique of combining lumbar plexus and sciatic nerve blocks with propofol sedation could alter the quality of recovery after TKR in elderly patients during the first week after surgery compared to general anesthesia. Our hypothesis was that patients who received combined lumbar plexus and sciatic nerve blocks supplemented with propofol sedation would have a higher incidence of recovery than patients who were receiving general anesthesia during the first week after surgery.

\section{Materials and methods}

The study was conducted at the Anesthesia and Operation Center of the Chinese People's Liberation Army General Hospital from June-September 2013. The research protocol was approved by the Institutional Ethical Committee of the Chinese People's Liberation Army General Hospital. After written, informed consent, 213 patients were enrolled.

\section{Selection of participants}

The inclusion criteria were patients who were: 65-90 years old; undergoing elective single TKR surgery; had met the American Society of Anesthesiologists' physical status I-III; had a Mini-Mental Score Examination (MMSE) $>23$; and had no an allergy to local or general anesthetics. The exclusion criteria included contraindications to nerve blocks (coagulation defects, infection at puncture site), current severe psychiatric disease, alcoholism or drug dependence, severe visual or auditory disorder, and unwillingness to return for study follow-up. ${ }^{10,11}$

\section{Interventions management}

General anesthesia was induced with midazolam $(0.015-0.03 \mathrm{mg} / \mathrm{kg})$, fentanyl $(1.8-3.5 \mu \mathrm{g} / \mathrm{kg})$, etomidate $(0.2-0.3 \mathrm{mg} / \mathrm{kg})$, and rocuronium $(0.4-0.6 \mathrm{mg} / \mathrm{kg})$, followed by a laryngeal mask airway insertion and mechanical ventilation. Anesthesia was maintained with a remifentanil infusion $(0.15-0.30 \mu \mathrm{g} / \mathrm{kg} / \mathrm{minute})$ and propofol (target controlled infusion [TCI] $0.6-2.0 \mu \mathrm{g} / \mathrm{mL}$ ) with $100 \%$ oxygen. Infusion rates of propofol and remifentanil varied, according to clinical judgment and a target bispectral index (BIS) range of 40-60.

Patients randomized to the peripheral nerve blocks group received midazolam $(0.015-0.03 \mathrm{mg} / \mathrm{kg})$ and fentanyl $(0.8-2.0 \mu \mathrm{g} / \mathrm{kg})$ titrated to provide conscious sedation before nerve block insertion. Supplemental oxygen ( $5 \mathrm{~L} /$ minute) was administered by facemask. After sterile preparation and draping, the nerve blocks were administered using a 21-gauge, $100 \mathrm{~mm}$ nerve block needle (Stimuplex $\mathrm{A}^{\circledR}$, B Braun Melsungen AG, Tochigi-ken, Japan) under nerve stimulator guidance. A posterior approach to lumbar plexus block was performed with patients in the lateral decubitus position. The puncture site was $3 \mathrm{~cm}$ caudad and $4 \mathrm{~cm}$ midline to the spinous process of L4. A sagittal insertion direction was used, and we avoided a medial angulation of the needle. Upon contact with transverse process of L5, the needle was retracted and then advanced over the transverse process $(2 \mathrm{~cm})$. The puncture depth was typically $6-10 \mathrm{~cm}$. The nerve stimulator was used to determine muscle activity from the femoral nerve (quadriceps or vastus lateralis). The puncture was also possible at the level of the transverse process of L4, with caudad advancement of the needle under the transverse process.

After a quadricep's muscle response had been identified with the nerve stimulator settings at $2 \mathrm{~Hz}$ frequency and current at $0.3-0.5 \mathrm{~mA}, 25-30 \mathrm{~mL}$ of $0.35 \%$ ropivacaine was injected in divided doses. ${ }^{12}$ A sciatic nerve block was performed in the same position after a twitch of hamstring, soleus, foot, or toes had been elicited using a similar current, and $15-25 \mathrm{~mL}$ of $0.35 \%$ ropivacaine was injected. Sensory and motor blocks on the operated limb were evalu- 
ated every 5 minutes after completion of the procedure until the achievement of adequate nerve block to allow surgery. A decision on the adequacy of the PNBs was made at 15 minutes after an insertion of the block. Sedation during surgery was provided by propofol (TCI $0.3-1.5 \mu \mathrm{g} / \mathrm{mL}$ ) with light sleep with easy arousal and a target BIS range of 60-80. Supplemental analgesia with 50-100 $\mu \mathrm{g}$ fentanyl was administered if required.

\section{Multimodal analgesic techniques}

Postoperative pain management included intravenous patient-controlled analgesia (sufentanil $1.25 \mu \mathrm{g} /$ hour as background dose and $1.25 \mu \mathrm{g}$ bolus with an 8 -minute lockout time), parecoxib (40 mg, every 12 hours), and oral oxycodone, $20 \mathrm{mg}$, was administered every 6 hours if required. Antiemetic tropisetron $2 \mathrm{mg}$ by intravenous infusion before surgery completion and then $0.1 \mathrm{mg} /$ hour intravenously until postoperative pain management finished.

\section{Surgical procedure}

To standardize recovery times and conditions, surgery was performed during the morning operating session. All operations were performed by two surgeons. A hemostatic tourniquet was always placed around the thigh and inflated to double the systolic arterial blood pressure.

\section{Postoperative recovery assessment}

The PQRS was administered to all participants by trained researchers. The researchers were blinded to the allocation. The questions assessing the ability to stand or walk of the ADL domain were not measured, due to the confounding effect of the knee surgery during the early recovery period. The PQRS has five assessment tools:

1. Orientation - name, date, and place (Adapted from the MMSE). Maximum score is 3.

2. Digits forward - assesses working memory (Adapted from the Wechsler Adult Intelligence Scale-111). Maximum score is 6 .

3. Digits backward - assesses working memory and scanning (Adapted from the Wechsler Adult Intelligence Scale-11l). Maximum score is 6.

4. Word recall - verbal memory word list (Adapted from the Rey Auditory Verbal Learning Test). Maximum score is 15 .

5. Word generation - tests executive function (Adapted from the Controlled Oral Word Association Test). Patients are given 30 seconds to say as many words as they can, starting with a given letter. No maximum score.
From pilot work, we had established that the word generation test was difficult for the elderly Chinese patients to comprehend, leading to low baseline scores. Previous research found that an assessment of orientation had shown a limited ability to discriminate between individuals. ${ }^{9}$ Accordingly, we made an a priori decision to exclude orientation and word generation in the cognitive domain. Baseline testing was performed 1-4 days preoperatively. The time zero $\left(\mathrm{T}_{0}\right)$ was defined as following the last skin stitch. The PQRS was measured at 15 minutes $\left(\mathrm{T}_{15}\right)$ and 40 minutes $\left(\mathrm{T}_{40}\right)$ after the operation and at 1 day, 3 days, and 7 days postoperatively, $\left(\mathrm{D}_{1}, \mathrm{D}_{3}\right.$, and $\left.\mathrm{D}_{7}\right)$.

Recovery is defined as "return to baseline values or better" at each time point. Change scores are calculated as the value at each time-baseline value, and the recovery is scored if the changes score $\geq 0$. The exception is scoring of recovery in the cognitive domain. Further validation of the PQRS, which occurred after the commencement of our study, has resulted in a recommendation to introduce an adjustment factor into the cognitive domain to account for performance variability. ${ }^{13}$ We used the new scoring proposed for cognitive recovery. In addition, patients were excluded from analysis in the cognitive domain (and all domains recovery) if their baseline scores on the cognitive questions were $\leq$ the adjustment factor, as low scores would automatically be scored as recovered. ${ }^{13}$ Other recovery domains, such as the nociception of emotive domains, use a 3-or 5-point Likert scale to measure the patient experience. The questions have both verbal as well as pictorial content to facilitate understanding across a larger age range. The full PQRS can be obtained from pqrsonline.org.

\section{Randomization and outcome measures}

Subjects were randomized in a 1:1 ratio to receive GA or PNBs. Consent was obtained by an investigator who did not participate in the surgery nor collect postoperative data. Randomization was performed using a computer-generated random numbers list using permuted blocks of size 4, created with StatMate ${ }^{\mathrm{TM}}$ v.1.01i software (GraphPad Software, Inc, La Jolla, CA, USA), and allocated by an investigator who was not involved in the surgery or the postoperative data collection. The PQRS surveys were conducted by investigators who were not involved in the conduct of surgery or anesthesia and were blinded to allocation. We excluded eight patients who had failed peripheral nerve blocks and required general anesthesia alone; postoperative data was not collected. We present a per protocol analysis. 
The primary outcome was quality of recovery in each domain measured with the PQRS, up to day 7. Secondary outcome measures were the consumption of opioid drugs, anesthesia time (defined as the duration of anesthesia or sedative drug administration), intraoperative blood pressure and heart rate, and the occurrence of side effects, such as the complications of PNBs.

\section{Sample size estimation}

Sample size estimates were performed using the Cochran-Mantel-Haenszel (CMH) approach and data from the PQRS study and our preliminary test. The study was powered to detect a moderate effect size (odds ratio $=3$ ), using a $\mathrm{CMH}$ test approach for all domains recovery (using nQueryAdvisor ${ }^{\circledR}$ 7.0, Statistical Solutions, Cork, Ireland), which required a minimal sample size of 85 per group. We adopted a conservative approach and increased the sample size to 110 per group to account for potential noncompletion of patients in this elderly cohort.

\section{Statistical analysis}

Categorical variables were presented as numbers and percentages, and continuous variables are presented as mean and standard deviation. The chi-square test and the Student's $t$-test were both used to investigate differences in patient characteristics and operative details between the two groups. Because of the risk of type I error with multiple comparisons, significance was defined as $<0.01$ for these measurements. Repeated measures analysis of variance was used to investigate the differences in the systolic blood pressure and the heart rate. Recovery domains of the PQRS were assessed by the CMH test, which assesses a global difference between groups over multiple time periods. For the global tests of repeated measures analysis of variance and $\mathrm{CMH}$, significance was defined as $P<0.05$.

\section{Results}

From June-September 2013, a total of 356 consecutive patients aged $\geq 65$ years old in our hospital were approached to participate in the study. Of these, 138 patients failed the inclusion criteria, did not consent to the study, or withdrew voluntarily prior to randomization, leaving 218 patients who completed the enrollment. Five patients were discharged $<7$ days after surgery and were lost to follow-up. Also, 213 patients were successfully completed in the analysis -108 patients in the GA group and 105 patients in the PNBs group (Figure 1).

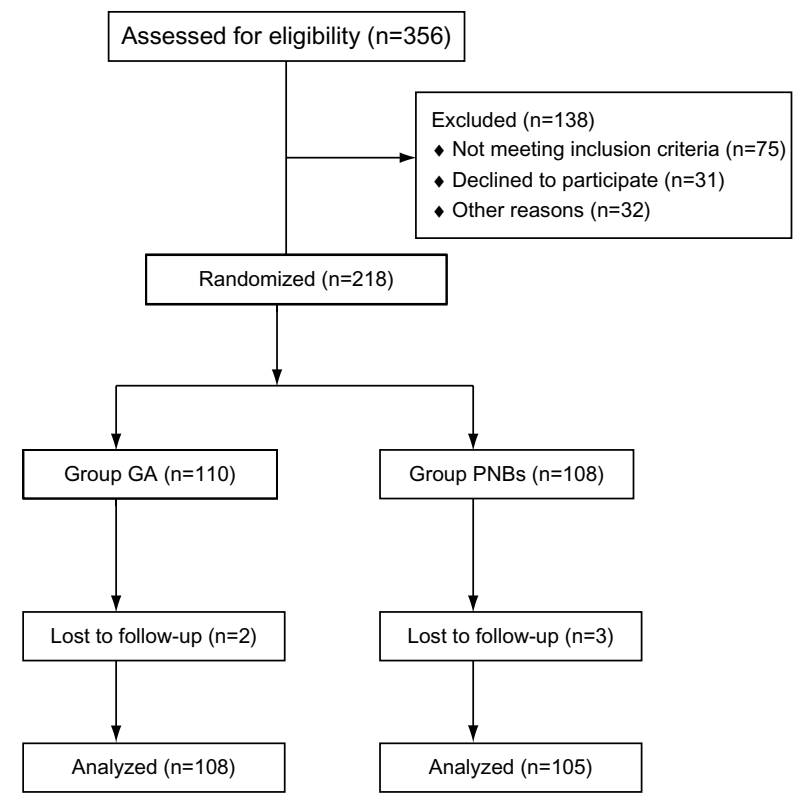

Figure I Flow chart showing patient enrollment, randomization, follow-up, and analysis.

Abbreviations: GA, general anesthesia; PNBs, peripheral nerve blocks.

Demographic data are shown in Table 1. There are no significant differences between the groups. The intraoperative variables are shown in Table 2. The anesthetic induction time of the PNBs group was longer $(20.1 \pm 7.4$ versus $8.4 \pm 2.5$ minutes, $P<0.001$ ), but it did not significantly alter total anesthetic time (162.3 \pm 26.8 versus $147.3 \pm 23.1$ minutes, $P=0.097$ ). The mean BIS was higher in the PNBs group ( $65 \pm 13.2$ versus $53 \pm 9.3$, $P<0.001)$. The intraoperative cumulative drug doses of the PNBs group were lower (all $P<0.001$ ) than the GA group.

Table I Demographic and preoperative data of the subjects

\begin{tabular}{|c|c|c|c|}
\hline & $\begin{array}{l}\text { GA group } \\
(n=108)\end{array}$ & $\begin{array}{l}\text { PNBs group } \\
(n=105)\end{array}$ & $P$-value \\
\hline Sex (male/female)* & $25(23.1 \%)$ & $23(21.9 \%)$ & 0.828 \\
\hline \multicolumn{4}{|l|}{ Age (year)* } \\
\hline $65-75$ & $68(63.0 \%)$ & 65 (6I.9\%) & 0.873 \\
\hline $76-85$ & $32(29.6 \%)$ & 31 (29.5\%) & 0.987 \\
\hline $86-89$ & $8(7.4 \%)$ & $9(8.6 \%)$ & 0.754 \\
\hline \multicolumn{4}{|l|}{ Education (year)* } \\
\hline $0-5$ & $23(21.3 \%)$ & $28(26.7 \%)$ & 0.359 \\
\hline $6-10$ & 69 (63.9\%) & $63(60.0 \%)$ & 0.559 \\
\hline$>11$ & $16(14.8 \%)$ & 14 (I3.3\%) & 0.756 \\
\hline Alcohol* & $16(14.8 \%)$ & $18(17.1 \%)$ & 0.643 \\
\hline Smoke* & $25(23.1 \%)$ & $22(21.0 \%)$ & 0.699 \\
\hline \multicolumn{4}{|l|}{ ASA status* } \\
\hline 1 & 7 (6.5\%) & $9(8.6 \%)$ & 0.563 \\
\hline 2 & $84(77.8 \%)$ & $78(74.3 \%)$ & 0.551 \\
\hline 3 & $17(15.7 \%)$ & $18(17.1 \%)$ & 0.783 \\
\hline
\end{tabular}

Note: Values are number (percentage), or mean \pm SD. $* \chi^{2}$ represents test of independence.

Abbreviations: ASA, American Society of Anesthesiologists; GA, general anesthesia; PNBs, peripheral nerve blocks; SD, standard deviation. 
Table 2 Intraoperative and postoperative data of subjects

\begin{tabular}{|c|c|c|c|}
\hline & $\begin{array}{l}\text { GA group } \\
(n=108)\end{array}$ & $\begin{array}{l}\text { PNBs group } \\
(n=105)\end{array}$ & $P$-value \\
\hline \multicolumn{4}{|l|}{ Intraoperation } \\
\hline $\begin{array}{l}\text { Anesthetic induction } \\
\text { time, minutes }\end{array}$ & $8.4 \pm 2.5$ & $20.1 \pm 7.4$ & $<0.00 \mathrm{I}$ \\
\hline Surgery time, minutes & $105.2 \pm 27.5$ & $106.9 \pm 21.7$ & 0.618 \\
\hline Anesthetic time, minutes & $|47.3 \pm 23|$. & $162.3 \pm 26.8$ & 0.097 \\
\hline Side of surgery, right/left & $69 / 49$ & $65 / 50$ & 0.763 \\
\hline Tourniquet, minutes & $92.2 \pm 20.4$ & $96.4 \pm 22.8$ & 0.158 \\
\hline Tourniquet, mmHg & $270 \pm 27$ & $275 \pm 23$ & 0.147 \\
\hline Midazolam, mg & $1.73 \pm 0.40$ & $1.30 \pm 0.5 \mathrm{I}$ & $<0.001$ \\
\hline Fentanyl, $\mu \mathrm{g}$ & $0.32 \pm 0.16$ & $0.07 \pm 0.03$ & $<0.001$ \\
\hline Propofol, mg & $359.5 \pm 143.8$ & $287.3 \pm 102.1$ & $<0.001$ \\
\hline Fluids administered, $\mathrm{mL}$ & $1,387 \pm 338$ & $\mathrm{I}, 442 \pm 280$ & 0.198 \\
\hline BIS & $52.6 \pm 9.3$ & $65.3 \pm 13.2$ & $<0.001$ \\
\hline \multicolumn{4}{|l|}{ Postoperation } \\
\hline \multicolumn{4}{|l|}{ Sufentanil consumption, $\mu \mathrm{g}$} \\
\hline 24 hours & $63.5 \pm 10.5$ & $37.5 \pm 8.5$ & $<0.001$ \\
\hline 72 hours & $147.0 \pm 22.5$ & $122.5 \pm 18.0$ & $<0.001$ \\
\hline
\end{tabular}

Notes: Values are mean $\pm S D$ or median. Two-tailed Student's $t$-test was used to compare groups. $P<0.01$ defined significance.

Abbreviations: GA, general anesthesia; PNBs, peripheral nerve blocks; BIS, bispectral index; SD, standard deviation.

The intraoperative systolic blood pressure was higher in the PNBs group $(P<0.001)$ as was the heart rate $(P<0.001)$, and it is shown in Figure 2. Physiological recovery was higher at $\mathrm{T}_{15}$ and $\mathrm{T}_{40}$, for the PNBs group, but equivalent by day $1(P<0.001)$.

Recovery in the nociceptive domain was higher with PNBs (Figure 3, $P<0.001$ ), though equivalence was reached by day 3 . The recovery profile for nausea and pain is shown in Figure 4 and shows improved recovery in both pain and nausea $(P<0.001)$ in the PNBs group, with equivalence reached by day 3 . The postoperative sufentanil requirement was lower in the PNBs during 24 hours and 72 hours (all $P<0.001$, Table 2).

Recovery was improved with PNBs in the emotive domain $(P<0.001)$ with better early recovery shown from both depression $(P=0.003)$ and anxiety $(P=0.008)$ questions (Figure 4$)$.

The modified ADL domain only included the assessment of eating and dressing and was not different between groups (Figure 3, $P=0.181$ ).

The modified cognitive domain was assessed using digits forward, digits back, and word recall questions only. Ten patients in the GA group and 12 patients in the PNBs group were excluded from analysis as their baseline scores were too low, being $\leq$ the adjustment factor for the cognitive tests, leaving 98 patients in GA group and 93 patients in PNBs group for analysis in the modified cognitive domain and all domains recovery. Recovery for the modified cognitive domain was better for PNBs (Figure 3, $P<0.001$ ) with equivalence reached by day 7 .

Recovery for all domains requires recovery in each of the physiological, emotive, nociceptive, cognitive, and ADL domains where applicable to the time period. The recovery of all domains was better for PNBs group (Figure $3, P<0.001$ ) with equivalence reached by day 7 .

The overall patient perspective domain is shown in Figure 5. This domain is not a recovery domain as it has no baseline measurement, but it aims to capture the patients' subjective assessment of their recovery. There was no difference between groups (all $P>0.05$ ). Satisfaction was high at all of the time points measured (exceeding 85\%) and was not different between the groups.
A Intraoperative systolic blood pressure

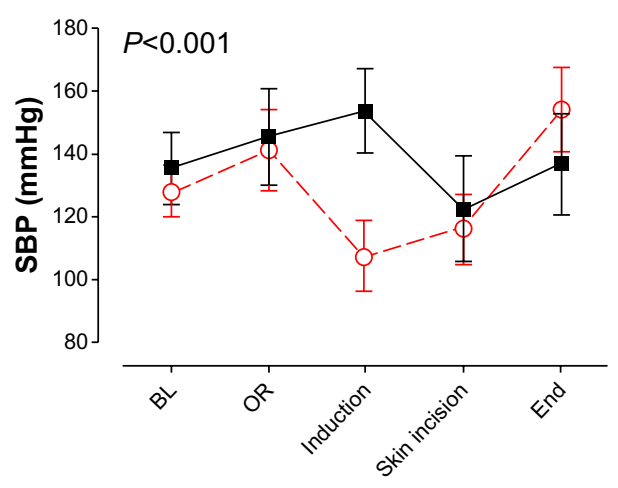

Measurement time points
B Intraoperative heart rate

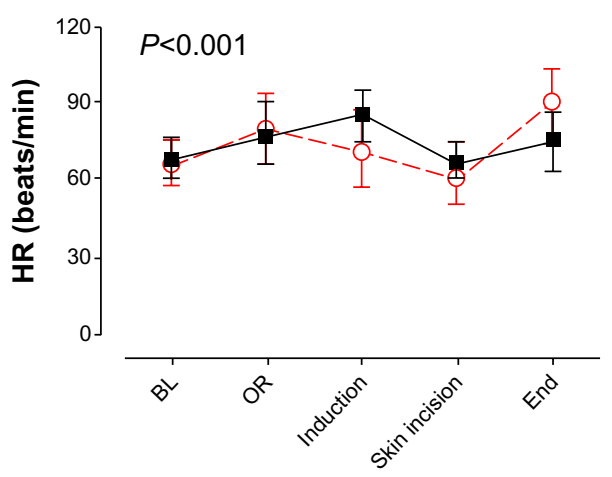

Measurement time points

Figure 2 Intraoperative systolic blood pressure and heart rate variation between the PNBs group $(\square)$ and the GA group $(O)$.

Notes: (A) intraoperative systolic blood pressure; (B) intraoperative heart rate.

Abbreviations: GA, general anesthesia; PNBs, peripheral nerve blocks; SBP, systolic blood pressure; HR, heart rate; BL, baseline (performed up to I-4 days presurgery); $\mathrm{OR}$, operation room; induction, anesthetic induction; skin incision, operation begin; end, end of surgery. 
A

Physiology domain

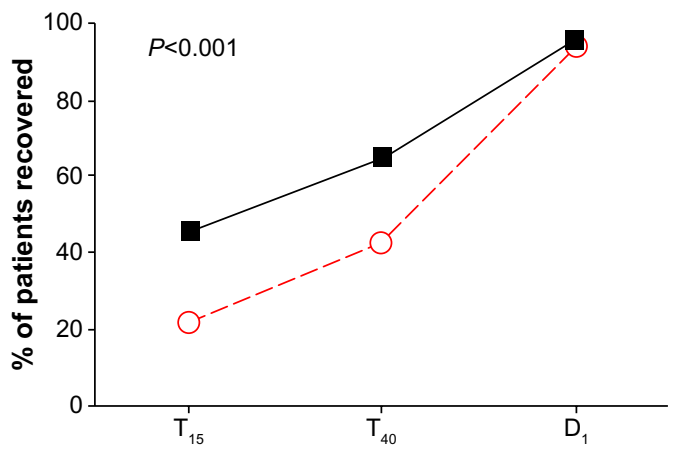

C

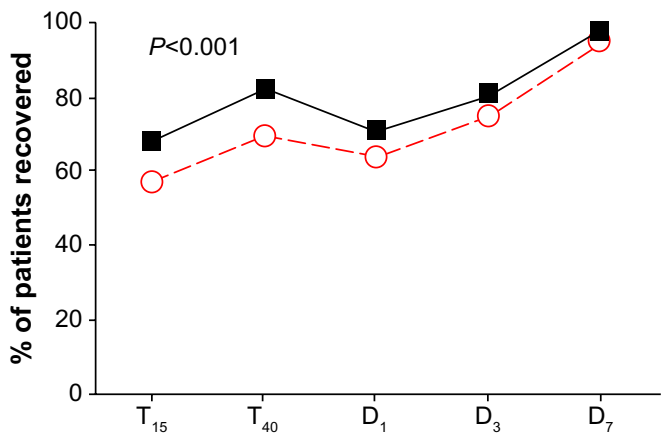

$\mathbf{E}$

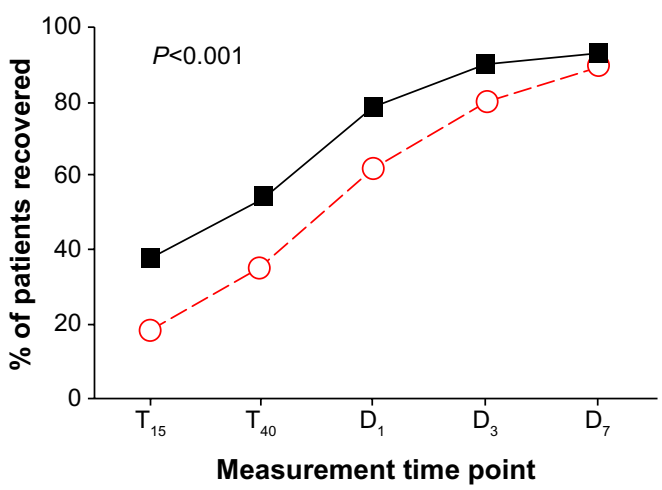

B

Modified ADL domain

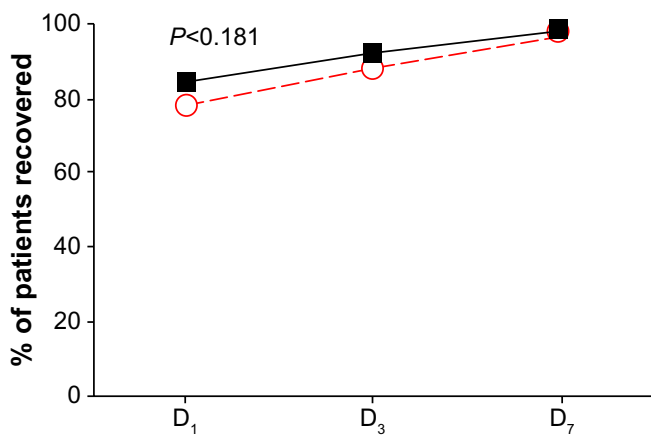

D

Nociceptive domain

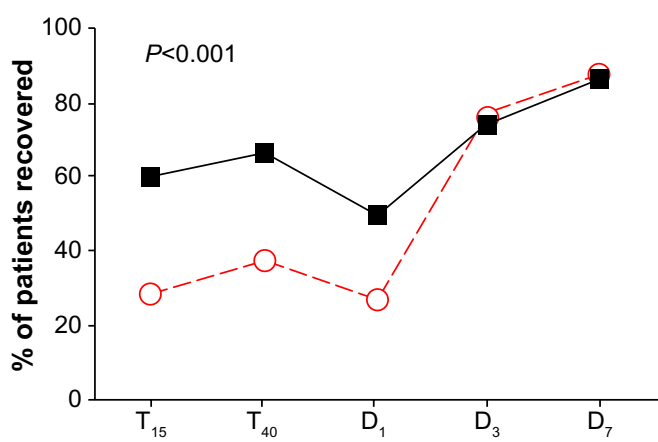

F

All domains recovery

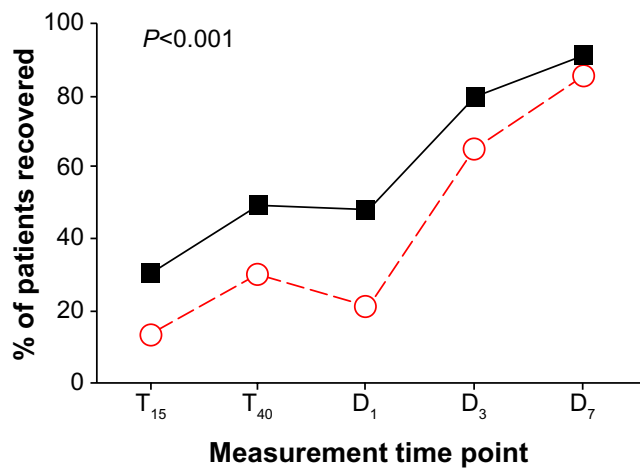

Figure 3 Postoperative recovery variation of the domains over time between the PNBs group $(\mathbf{\square})$ and the GA group (O).

Notes: The domains include (A) physiology, (B) modified ADL, (C) emotive, (D) nociceptive, (E) modified cognitive, and (F) all domains recovery.

Abbreviations: GA, general anesthesia; PNBs, peripheral nerve blocks; $A D L$, activities of daily living; $B L$, baseline; $T_{15}, I_{15}$ minutes after surgery; $T_{40}, 40$ minutes after surgery; $D_{1}$, I day after surgery; $D_{3}, 3$ days after surgery; $D_{7}, 7$, days after surgery.

\section{Discussion}

This study demonstrated that patients over the age of 65 years undergoing TKR achieved a high proportion of recovery in all domains 1 week after surgery. The PNBs, as the primary anesthetic technique supplemented by propofol sedation and compared to the general anesthesia consisting of remifentanil and propofol, were associated with a faster recovery in all domains other than ADL, less fluctuant intraoperative blood pressure and heart rate, and lower postoperative opioid consumption. No differences in recovery were found between day 3 and day 7 for different assessment domains.

The measurement of quality of recovery represents a paradigm shift for anesthesia and surgery practice, as it changes the focus on outcomes from hospital-based (morbidity, mortality, 
A

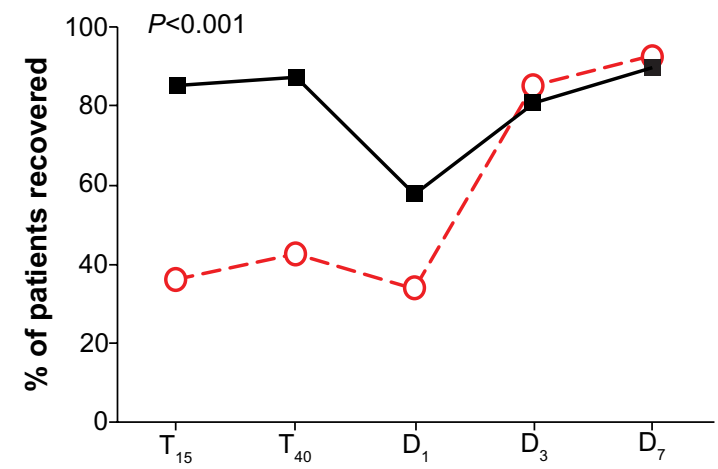

C

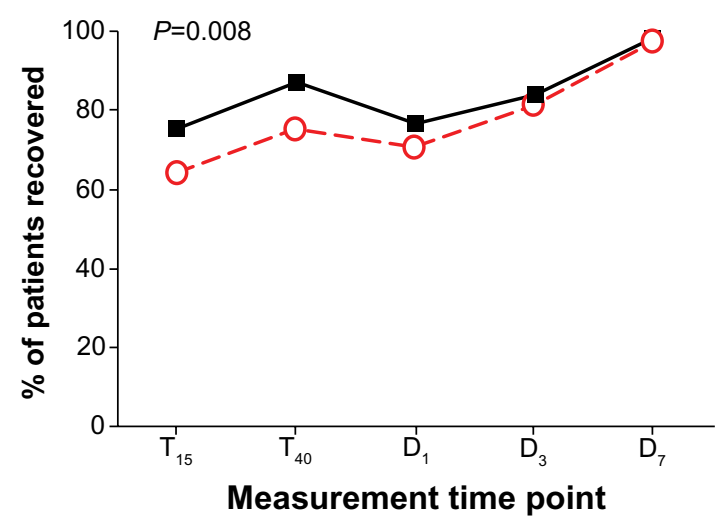

B

Nociception - nausea

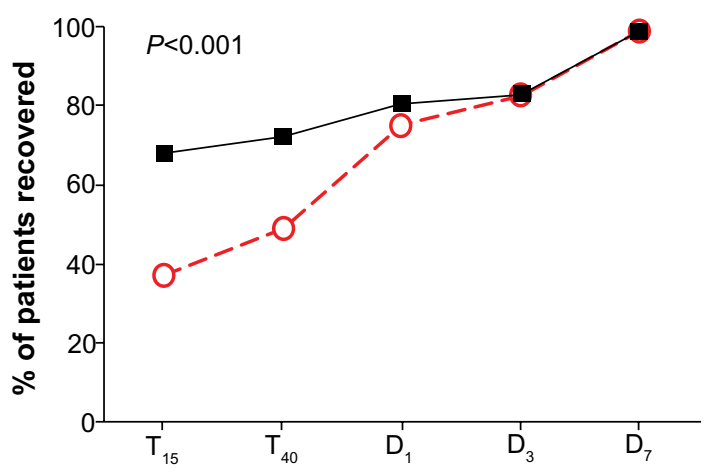

D

Emotion - depression

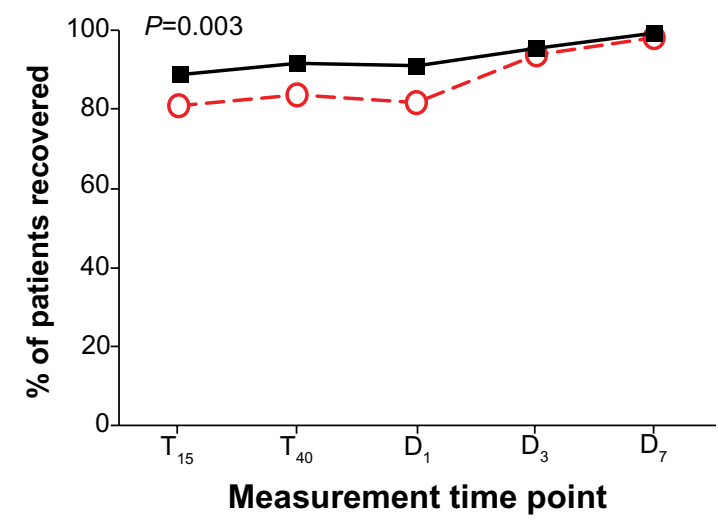

Figure 4 Incidence of full recovery between the PNBs group $(\square)$ and the GA group $(O)$ in nociceptive and emotive domain.

Notes: The nociceptive domain includes $(\mathbf{A})$ pain and $(\mathbf{B})$ nausea. Emotive domain includes $(\mathbf{C})$ anxiety and $(\mathbf{D})$ depression. Zero indicated failure of recovery. The maximum number in each graph denotes full recovery.

Abbreviations: GA, general anesthesia; PNBs, peripheral nerve blocks; $B L$, baseline; $T_{15}, 15$ minutes after surgery; $T_{40}, 40$ minutes after surgery, $D_{1}, I$ day after surgery; $D_{3}, 3$ days after surgery; and $D_{7}, 7$ days after surgery.

and length of stay), to patient-focused outcomes. For anesthesiologists, this represents a concept that the techniques used in the operating room may impact on recovery long after the physiological and pharmacological effects of the anesthetic have diminished. Although the use of the PNBs predominantly affects the pain response in the first few hours after surgery, they also reduce the exposure to sedative or general anesthetic drugs, which - in turn - could have a longer-term impact on recovery. The PQRS is a measurement tool that was purposely designed to look at a broad range of recovery domains in an objective fashion over repeated time periods to identify the impact of surgery and anesthesia on recovery in multiple different aspects or domains of recovery. This included cognition, because it is increasingly being recognized as a major source of morbidity for patients, even though they have physically recovered well.

The use of regional anesthesia is common in the People's Republic of China, but it is not universally adopted as a preferred technique worldwide. There is always a debate about the risk/benefit ratio of using regional anesthesia. Regional anesthesia imposes risks, such as nerve damage and local anesthetic toxicity. The total ropivacaine dose of nerve blocks in this study did not exceed $4 \mathrm{mg} / \mathrm{kg}$. There were no instances of symptoms consistent with local anesthetic toxicity in the perioperative period. There was one minor neuropraxia which resolved over 3 weeks, but as upper leg tourniquets were used, we cannot be totally confident that it was solely due to the nerve block. In our hands, the technique was effective for providing surgical anesthesia. However, general anesthesia is not without risk, and the increased exposure to anesthetic drugs could incur risk, including poorer patient recovery. When comparing techniques, a risk-risk analysis is perhaps better than individually assessing risk-benefit analysis for individual techniques. Our data do not allow us to define the relative risks of the two techniques, other than to state that both techniques were associated with low morbidity. A meta-analysis compared regional to general anesthesia for 


\section{A Self-assessment, ADL: (eat and dress)}

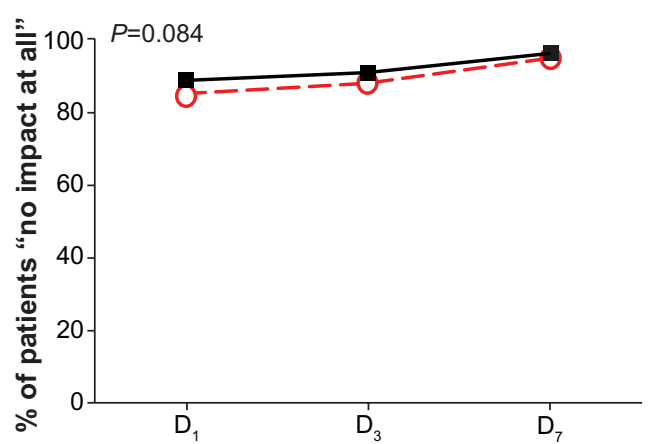

\section{B}

Self-assessment, clarity of thought

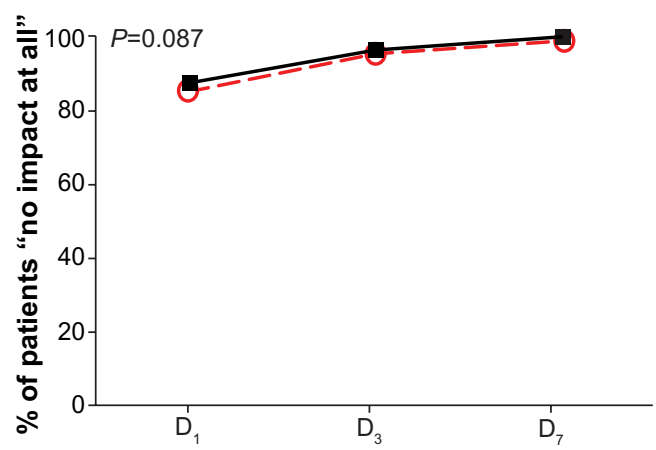

C

Self-assessment, satisfaction

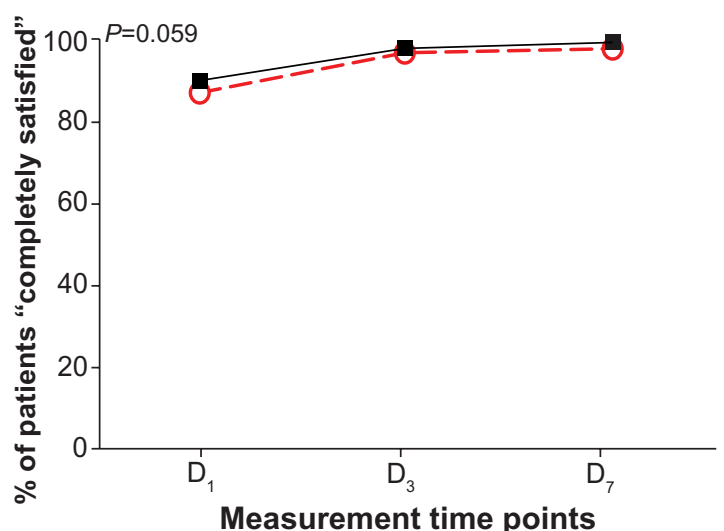

Figure 5 Percentage of patients who rated their overall perspective of the operative procedure between the PNBs group $(\boldsymbol{\square})$ and the GA group $(O)$.

Notes: The domain includes (A) ADL (eat and dress), (B) clarity of thought, and (C) satisfaction. Also, 100\% indicates patients feel "no impact at all".

Abbreviations: GA, general anesthesia; PNBs, peripheral nerve blocks; ADL, activities of daily living; $D_{1}, I$ day after surgery; $D_{3}, 3$ days after surgery; $D_{7}, 7$ days after surgery.

lower limb joint surgery in 2009. ${ }^{6}$ Strong conclusions were unable to be drawn due to the limited number of randomized studies and the larger variation in endpoints to define full or poor recovery. However, there was a trend toward poorer cognitive outcomes in patients who received only general anesthesia. Further, most studies were conducted in a Western population, rather than a broad representation of countries, racial groups, and the differences in techniques within these countries. Our study adds to the literature in this area by using a multidimensional, objective recovery assessment tool as well as defining recovery in an elderly Chinese population.

Postoperative pain associated with TKR is considerable and requires adequate analgesia. ${ }^{14,15}$ The single-injection of PNBs provided 12-24 hours of analgesia in our study, which is similar to the previous report. ${ }^{16}$ The pain recovery was markedly different at the 15-minute and the 40-minute time periods between the two groups. Though the incidence of recovery for pain decreased from 40 minutes to 1 day in the PNBs group, the pain recovery was still higher than the GA group at 1 day, suggesting the presence of residual analgesia from nerve blocks in a proportion of patients. By day 3, there was equivalence between the groups. Nausea was lower with PNBs in the early time periods only, which may be, in part, due to an improved pain relief as well as the lower consumption of opioid analgesia. ${ }^{17,18}$

The emotive recovery was faster in both anxiety and depression questions in the PNBs group, although equivalence was achieved by day 3 . We cannot determine whether this effect was due to improved analgesia or a lower consumption of anesthesia and analgesia medication. Further, emotive recovery is a complex area; it can involve preoperative expectation and the hospital environment, including altered sleep patterns, which may be more pronounced in the elderly. ${ }^{19}$

The modified ADL domain only includes the ability to eat and to dress due to the limitation imposed by surgery. In these two items, recovery was high and not different between the groups.

Postoperative cognitive recovery is a very important area of research and has been studied extensively. The literature is confusing, however, due to the many different tests and the definitions used to measure cognitive decline. Many elderly patients suffer significant deterioration of cognitive function after cardiac surgery ${ }^{20}$ or noncardiac major surgery, ${ }^{10,21}$ even after seemingly uneventful procedures. The PQRS assesses recovery, taking a different approach in that it assesses cognitive recovery, rather than the postoperative cognitive deficit (POCD), ${ }^{22,23}$ although there is likely to be an association with failure to recover and POCD. Further, the PQRS does not assess delirium, which is a fluctuating mental state, ${ }^{23}$ although it is likely that delirious patients will be less likely to show recovery. The definition of cognitive recovery has 
been amended from the original PQRS publication after the validation of the test accuracy in normal volunteers not undergoing surgery. ${ }^{13}$ Normal volunteers will have variability in cognitive performance based on the time of day. Accordingly, an adjustment factor is applied to each test, such that patients can perform a little worse than baseline and still be coded as recovered. The recommendation is that recovery rates exceeding $80 \%$ should be considered in a range of "very good recovery". ${ }^{13}$

Further, the cognitive recovery in our study is not equivalent to other PQRS publications as we modified the scale a priori. Although this is a limitation of our study, the results for the remaining cognitive tests showed a faster cognitive recovery in the PNBs group, with equivalence reached by day 7. We cannot determine from our data the exact reason for this, but rather we can identify that the specific anesthesia and analgesia protocol with the PNBs resulted in faster cognitive recovery than the specific general anesthesia and analgesia regimen in our comparative group. We urge caution not to extrapolate our findings to regional anesthesia versus general anesthesia, as there may be marked differences in the various regional anesthesia techniques, and for different combinations of anesthetic drugs.

Our study only assessed recovery to day 7 , and we cannot determine if the failure to recovery by day 7 is linked to longer-term recovery. In a study of cardiac surgery patients, Royse et al showed lower POCD at 3-5 days with propofol versus desflurane, but this was not associated with a difference in POCD at 3 months after surgery. ${ }^{24}$ More research is required to identify whether failure to recover in the PQRS in the first week after surgery is a harbinger of POCD.

When assessing complete recovery (recovery in all domains), the PNBs group recovered faster than the GA group with equivalence reached by day 7 . This is not surprising, given the differences in all domains except ADL.

The overall perspective domain is not a recovery domain as there is no baseline measurement. However, it can capture the patient self-assessment including satisfaction. Patients always underestimate their cognitive performance compared with objective testing, and this effect was shown in the original PQRS publication, ${ }^{9}$ emphasizing the importance of objective testing of cognition. Similar to other data, satisfaction was high in both groups and not different. This is a consistent finding and emphasizes that satisfaction is a poor discriminator of quality of recovery. ${ }^{8}$

There are limitations to our study. A number of patients were excluded from the cognitive recovery analysis, because they had very low baseline values. In the PQRS, a tolerance factor was applied, such that the patients can be a little worse and still score as recovered, due to the normal performance variability for cognitive performance. If the baseline score is $\leq$ to the adjustment to the score, then they would automatically score as "recovered," and the test would lose discriminant ability to measure poor cognitive recovery in these patients. ${ }^{13}$ We reduced this risk by first screening with MMSE and excluded patients with scores of $<24$. However, the MMSE is primarily aimed at detecting dementia, ${ }^{25}$ and so we post hoc applied the recommendation of Royse et al ${ }^{13}$ to exclude patients from the cognitive analysis when their baseline scores were $\leq$ to the correction factor for the tests. The modification of the ADL and cognitive domains does limit conclusions about recovery to our cohort and is not directly comparable to other PQRS publications. The randomized trial design, however, does allow us to directly compare our two groups. We were concerned that anxiety and concerns about the forthcoming operation can affect the baseline value ${ }^{26}$ and so conducted most baseline assessments at least 1 day prior to surgery. To reduce the assessor bias, the researchers conducting the PQRS were blinded to the allocation. It is not possible, however, to absolutely assure concealment as patients with a successful nerve block may have an absence of pain and leg weakness early after surgery. However, this is a potential bias that we cannot control. The anesthetic techniques used in this study, as well as the postperative management including physical therapy, though commonly used in our hospital, may be different to other centers in the Western world. We cannot comment on whether our techniques could impact on hospital resource use or the ability to fast track patients to discharge.

\section{Conclusion}

Lumbar plexus and sciatic blocks with sedation facilitates faster postoperative recovery than general anesthesia, but not at 1 week, after TKR in patients 65 years or older. This provides proof of concept that the use of regional anesthesia could impact on quality outcomes.

\section{Acknowledgments}

The authors thank the many research staff, nursing staff, and surgical and anesthesiology colleagues who have helped with the conduct of the study in the Chinese People's Liberation Army General Hospital. In particular, the authors thank Yonggang Zhou, MD, $\mathrm{PhD}$, and professor in the Department of Orthopaedics, Chinese People's Liberation Army General Hospital, Beijing, People's Republic of China, and Jiying 
Chen, MD, PhD, and professor in the Department of Orthopaedics, the Chinese People's Liberation Army General Hospital, Beijing, People Republic of China.

\section{Author contributions}

JunLe Liu helped design the study and wrote the manuscript; WeiXiu Yuan and XiaoLin Wang collected the original data and performed the analysis; Colin F Royse is the organizer of the Postoperative Quality of Recovery Scale, participated in the trial design, supervised the trial, and revised the manuscript critically. MaoWei Gong and Ying Zhao collected the original data and were involved with writing the paper; Hong Zhang was responsible for conception and management of the study and audit design, edited the manuscript, and is guarantor. All authors approved of the version to be published. The authors agreed to be accountable for all aspects of the work in ensuring that questions related to the accuracy or integrity of any part of the work are appropriately investigated and resolved.

\section{Disclosure}

The authors report no conflicts of interest in this work.

\section{References}

1. Carr AJ, Robertsson O, Graves S, et al. Knee replacement. Lancet. 2012;379(9823):1331-1340.

2. Manku K, Bacchetti P, Leung JM. Prognostic significance of postoperative in-hospital complications in elderly patients. I. Long-term survival. Anesth Analg. 2003;96(2):583-589, table of contents.

3. Young A, Buvanendran A. Recent advances in multimodal analgesia. Anesthesiol Clin. 2012;30(1):91-100.

4. Stevens RD, Van Gessel E, Flory N, Fournier R, Gamulin Z. Lumbar plexus block reduces pain and blood loss associated with total hip arthroplasty. Anesthesiology. 2000;93(1):115-121.

5. Capdevila X, Macaire P, Dadure C, et al. Continuous psoas compartment block for postoperative analgesia after total hip arthroplasty: new landmarks, technical guidelines, and clinical evaluation. Anesth Analg. 2002;94(6):1606-1613, table of contents.

6. Hu S, Zhang ZY, Hua YQ, Li J, Cai ZD. A comparison of regional and general anaesthesia for total replacement of the hip or knee: a meta-analysis. J Bone Joint Surg Br. 2009;91(7):935-942.

7. Hadzic A, Karaca PE, Hobeika P, et al. Peripheral nerve blocks result in superior recovery profile compared with general anesthesia in outpatient knee arthroscopy. Anesth Analg. 2005;100(4):976-981.

8. Royse CF, Chung F, Newman S, Stygall J, Wilkinson DJ. Predictors of patient satisfaction with anaesthesia and surgery care: a cohort study using the Postoperative Quality of Recovery Scale. Eur J Anaesthesiol. 2013;30(3):106-110.
9. Royse CF, Newman S, Chung F, et al. Development and feasibility of a scale to assess postoperative recovery: the post-operative quality recovery scale. Anesthesiology. 2010;113(4):892-905.

10. Moller JT, Cluitmans P, Rasmussen LS, et al. Long-term postoperative cognitive dysfunction in the elderly ISPOCD1 study. ISPOCD investigators. International Study of Post-Operative Cognitive Dysfunction. Lancet. 1998;351(9106):857-861.

11. Monk TG, Weldon BC, Garvan CW, et al. Predictors of cognitive dysfunction after major noncardiac surgery. Anesthesiology. 2008;108(1): $18-30$.

12. Chayen D, Nathan H, Chayen M. The psoas compartment block. Anesthesiology. 1976;45(1):95-99.

13. Royse CF, Newman S, Williams Z, Wilkinson DJ. A human volunteer study to identify variability in performance in the cognitive domain of the postoperative quality of recovery scale. Anesthesiology. 2013;119(3):576-581.

14. Pavlin DJ, Chen C, Penaloza DA, Polissar NL, Buckley FP. Pain as a factor complicating recovery and discharge after ambulatory surgery. Anesth Analg. 2002;95(3):627-634.

15. Wu CL, Rowlingson AJ, Partin AW, et al. Correlation of postoperative pain to quality of recovery in the immediate postoperative period. Reg Anesth Pain Med. 2005;30(6):516-522.

16. Hadzic A, Williams BA, Karaca PE, et al. For outpatient rotator cuff surgery, nerve block anesthesia provides superior same-day recovery over general anesthesia. Anesthesiology. 2005;102(5):1001-1007.

17. van den Bosch JE, Bonsel GJ, Moons KG, Kalkman CJ. Effect of postoperative experiences on willingness to pay to avoid postoperative pain, nausea, and vomiting. Anesthesiology. 2006;104(5):1033-1039.

18. Sinclair DR, Chung F, Mezei G. Can postoperative nausea and vomiting be predicted? Anesthesiology. 1999;91(1):109-118.

19. Gustafsson BA, Ponzer S, Heikkilä K, Ekman SL. The lived body and the perioperative period in replacement surgery: older people's experiences. $J$ Adv Nurs. 2007;60(1):20-28.

20. Bedford PD. Adverse cerebral effects of anaesthesia on old people. Lancet. 1955;269(6884):259-263.

21. Newman S, Stygall J, Hirani S, Shaefi S, Maze M. Postoperative cognitive dysfunction after noncardiac surgery: a systematic review. Anesthesiology. 2007;106(3):572-590.

22. Murkin JM, Newman SP, Stump DA, Blumenthal JA. Statement of consensus on assessment of neurobehavioral outcomes after cardiac surgery. Ann Thorac Surg. 1995;59(5):1289-1295.

23. Robinson TN, Raeburn CD, Tran ZV, Angles EM, Brenner LA, Moss M. Postoperative delirium in the elderly: risk factors and outcomes. Ann Surg. 2009;249(1):173-178.

24. Royse CF, Andrews DT, Newman SN, et al. The influence of propofol or desflurane on postoperative cognitive dysfunction in patients undergoing coronary artery bypass surgery. Anaesthesia. 2011;66(6): 455-464.

25. Ghoneim MM, Block RI. Clinical, methodological and theoretical issues in the assessment of cognition after anaesthesia and surgery: a review. Eur J Anaesthesiol. 2012;29(9):409-422.

26. Funder KS, Steinmetz J, Rasmussen LS. Methodological issues of postoperative cognitive dysfunction research. Semin Cardiothoracic Vasc Anesth. 2010;14(2):119-122.
Clinical Interventions in Aging

\section{Publish your work in this journal}

Clinical Interventions in Aging is an international, peer-reviewed journal focusing on evidence-based reports on the value or lack thereof of treatments intended to prevent or delay the onset of maladaptive correlates of aging in human beings. This journal is indexed on PubMed Central, MedLine, the American Chemical Society's 'Chemical Abstracts

\section{Dovepress}

Service' (CAS), Scopus and the Elsevier Bibliographic databases. The manuscript management system is completely online and includes a very quick and fair peer-review system, which is all easy to use. Visit http://www.dovepress.com/testimonials.php to read real quotes from published authors. 